exclusion of 3 included the two with previous small endoleak. However, one who had complete exclusion immediately recur the filling during follow-up. This patient treated with re-dilation of the stent using balloon. Final follow-up angiography showed complete exclusion of all CCFs and revealed good stent patency of the ICA without intra-stent stenosis.

Conclusion Graft-stents should be considered as an alternative option of treating CCFs and preserving the parent artery by arterial wall reconstruction especially in patients with a fistula that cannot be successfully occluded with detachable balloons or coils. Although a larger sample and expanded follow-up are needed, our series shows that covered stents can be used in the treatment of CCFs with symptomatic relief as experience.

Disclosures S. Chung: None. S. Chang: None.

\section{E-069 SUPER-SELECTIVE FISTULA POINT COIL EMBOLIZATION FOR CAROTID-CAVERNOUS ARTERIOVENOUS FISTULA}

S Majidi ${ }^{*}$, I Singh, J Fifi. Neurosurgery, Mount Sinai Hospital, New York, NY

\subsection{6/neurintsurg-2019-SNIS.144}

Background/Purpose Endovascular treatment of carotid-cavernous fistula (CCF) has evolved over the past two decades from using detachable balloons to Onyx and coil embolization. Nowadays, transvenous coil embolization is the mainstay of CCF management. Obliteration of the cavernous sinus with coils or liquid embolic agent can be time consuming and costly and potentially lead to cranial nerve injury. We report technical aspects and clinical outcome of a transvenous superselective fistula point coil embolization.

Methods We retrospectively reviewed consecutive patients with CCF who were treated by a single operator in our institution from January 2016 to December 2018. Patients' demographics, type of CCF, endovascular embolization technique and clinical and angiographic outcome were analyzed. Super-selective fistula point embolization is a technique by which microcatheter exploration allows for definition of the fistula location within the cavernous sinus and targeted coiling at this point.

Results A total of 11 patients were identified. All patients had type D dural arteriovenous CCF. Fifty percent of the patients were female. The mean age $( \pm S D)$ of the patients were 61 \pm 13 years. All patients were treated with transvenous superselective coil embolization of the fistula point (figure-1). The inferior petrosal sinus was used to access the fistula point at the cavernous sinus and superior ophthalmic vein junction in 9 patients. In the remaining 2 patients, the facial vein was used to access the fistula point. Ten patients had complete fistula obliteration in the final angiographic run at the end of the embolization procedure. One patient had small residual fistula which was completely obliterated by further super-selective coiling during follow up angiography. No procedure related morbidity or mortality was observed. All 11 patients had complete resolution of their clinical symptoms. A total of 7 patients had follow up cerebral angiography and 3 patients had follow up MRI/MRA at the 6 to 12 month timepoint which were all indicative of complete obliteration of the CCF. Conclusions Super-selective transvenous coil embolization of a focal fistula point in patients with CCF is safe and feasible and is associated with excellent durable clinical and angiographic outcome.

Disclosures S. Majidi: None. I. Singh: None. J. Fifi: None.

\section{$\mathrm{E}-070$} CURATIVE TREATMENT FOR LOW GRADE ARTERIOVENOUS MALFORMATIONS

${ }^{1} \mathrm{~A}$ Wang*, ${ }^{2} \mathrm{E}$ Connolly, ${ }^{1} \mathrm{R}$ Solomon, ${ }^{1} \mathrm{~S}$ Lavine, ${ }^{1} \mathrm{P}$ Meyers. ${ }^{1}$ Neurosurgery/Radiology, Columbia University Medical Center, New York, NY; ${ }^{2}$ Neurosurgery, Columbia University Medical Center, New York, NY

10.1136/neurintsurg-2019-SNIS. 145

Introduction Low grade, Spetzler Martin (SM) grade I and II arteriovenous malformations (AVM) are often considered safe for surgical resection or radiosurgery. The use of preoperative arteriography with endovascular embolization to reduce surgical risk in these AVMs remains controversial. The authors assessed the safety of combined treatment of SM I and II AVMs with preoperative embolization followed by curative treatment with surgical resection or radiosurgery. Long term functional outcomes were also studied.

Methods Under IRB-approved protocol, retrospective analysis was carried out on all patients with ruptured and unruptured SM I and II AVMs from 2002 to 2017 using a quality assurance database. Details of the endovascular procedures including arterial supply to the AVM, number of branches embolized, embolic agent(s) used, and complications were studied. Baseline clinical and imaging characteristics were compared, and functional status using the modified Rankin Scale (mRS) before and after endovascular and microsurgical/radiosurgery treatments were compared.

Results 258 SM I (36\%) and II (64\%) AVMs were identified (mean age 38.3 years). $48 \%$ of patients presented with hemorrhage, $21 \%$ with seizure, $16 \%$ with headache, $10 \%$ asymptomatic and 5\% with a clinical deficit. 90 patients $(68 \%)$ in the unruptured group and 74 patients (59\%) in the ruptured group underwent presurgical embolization $(p=0.0013)$. The mean number of arteries supplying the AVM was 1.44 and 1.41 in the unruptured and ruptured groups respectively. The mean number of arteries embolized was 2.51 in the unruptured group compared to 1.8 in the ruptured group $(\mathrm{p}=$ 0.003). nBCA and Onyx were the two most commonly used embolic agents. Pre-embolization and post-embolization mRS are shown in tables 1 and 2. Four complications were seen in

Abstract E-070 Table 1 Preoperative embolization outcome in low grade unruptured AVMs

\begin{tabular}{lll}
\hline Overall mRS (\%) & Pre-embolization & Post-embolization \\
\hline $0-2$ & $90(100 \%)$ & $90(100 \%)$ \\
3 & 0 & 0 \\
4 & 0 & 0 \\
5 & 0 & 0 \\
6 & 0 & 0 \\
\hline
\end{tabular}

Abstract E-070 Table 2 Preoperative embolization outcome in low grade ruptured AVMs

\begin{tabular}{lll}
\hline Overall mRS (\%) & Pre-embolization & Post-embolization \\
\hline $0-2$ & $64(93.9 \%)$ & $63(93.3 \%)$ \\
3 & $7(4.3 \%)$ & $7(4.3 \%)$ \\
4 & $3(1.8 \%)$ & $4(2.4 \%)$ \\
5 & 0 & 0 \\
6 & 0 & 0 \\
\hline
\end{tabular}


164 embolization procedures including two peri/post procedural hemorrhages, one dissection, and one infarct. All patients undergoing microsurgical resection had a complete cure on post-operative angiography. Immediate postoperative mRS and long term follow up mRS (mean of 55 months) was determined after curative treatment. Good long term outcomes (mRS $\leq 2)$ was seen in $95 \%$ of unruptured AVM patients and 92\% of ruptured AVM patients postoperatively. Transient and permanent neurological deficits were seen in 5\% and $1 \%$ of patients after curative treatment.

Conclusions Multimodal therapy of low grade AVMs can be performed with low morbidity and high cure rates in high volume centers. By comparison with other published studies, our outcomes suggest that pre-operative embolization is a safe adjunct to definitive curative treatment.

Disclosures A. Wang: None. E. Connolly: None. R. Solomon: None. S. Lavine: None. P. Meyers: None.

\section{E-071 PERCUTANEOUS ALCOHOL EMBOLIZATION OF SYMPTOMATIC VERTEBRAL HEMANGIOMA USING COLUMN TECHNIQUE: A CASE REPORT}

S Male*, T Mehta, A Khan, A Grande, R Tummala, B Jagadeesan. University of Minnesota, Minneapolis, MN

\subsection{6/neurintsurg-2019-SNIS. 146}

Background Hemangiomas are the most common vertebral body tumors typically discovered as incidental findings on spinal imaging. In $1 \%$ of patients, hemangiomas can be symptomatic causing persistent back pain that necessitate treatment. Percutaneous alcohol embolization (PAE) is an effective but challenging way of treating these lesions. We hereby report a case of vertebral hemangioma treated with PAE with unique technique.

Methods 59-year-old man with chronic back pain refractory to medications, physical therapy and chiropractic maneuvers presented to our clinic. His MRI spine revealed multiple vertebral hemangiomas, most prominent at T8 and T10. On examination, the point of maximum tenderness was at T10. Limited spinal angiogram revealed tumor blush at the sites of vertebral hemangiomas. Under sterile conditions, two 10-gauge needles were advanced in the T10 vertebral body via transpedicular approach. The contrast injection revealed tumor blush and venous outflow into the azygous system. For PAE, we formed columns of contrast-absolute ethanol-contrast to avoid alcohol dilution and for adequate visualization during embolization. (figure 1) A bubble trapped between these columns, initially

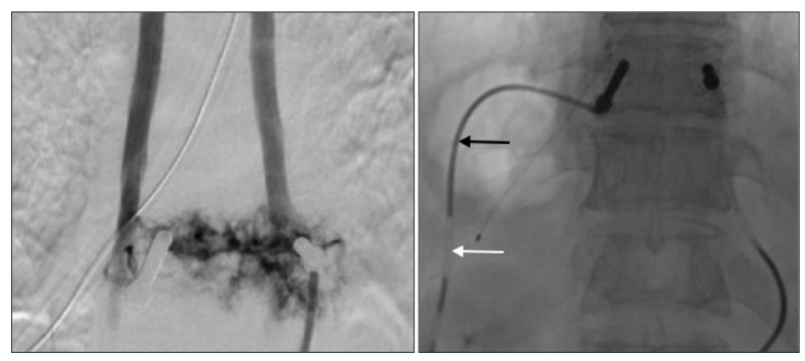

Abstract E-071 Figure 1 inadvertently and later intentional, also helped to keep the columns separate. The mixing of contrast to absolute ethanol is an alternative to this technique. However, it could decrease the efficacy of absolute ethanol in obliteration of hemangioma. Patient tolerated the procedure very well and endorsed clinical improvement on day 1.

Conclusion During PAE for vertebral hemangiomas, formation of columns of contrast and absolute ethanol during embolization is an effective technique. It helps to preserve higher alcohol concentration and in turn, efficacy of embolization while maintaining adequate visualization.

Disclosures S. Male: None. T. Mehta: None. A. Khan: None. A. Grande: None. R. Tummala: None. B. Jagadeesan: None.

\section{E-072 IMPACT OF OBESITY ON SHORT-TERM IN-HOSPITAL OUTCOMES IN PATIENTS UNDERGOING SPINAL FUSION PROCEDURES}

T Colburn*, D Schirmer, Z Rose-Reneau, B Wright. Kansas City University of Medicine and Biosciences, Kansas City, MO

10.1136/neurintsurg-2019-SNIS.147

Background Spinal fusion is a surgical procedure utilized to treat or alleviate several spinal diagnoses including: tumor, spinal stenosis, degenerative disc disease, scoliosis, and spondylolisthesis. Previous studies have explored clinical outcomes for this intervention. This study aims to further investigate shortterm outcomes of patients after spinal fusion by looking at the impact of obesity.

Methods This retrospective cohort study utilized data from the Nationwide Inpatient Sample (NIS) to identify adult patients $(18+)$ from 2012-2015 who underwent spinal fusion. ICD-9 codes identified these patients; specifically patients diagnosed with obesity. Any patients missing important clinical identifiers (age, gender, cause of death) and patients without spinal fusion intervention were excluded. Data analyses assessed hospital length of stay (LOS), inpatient charges, average age of admission and mortality rate.

Results Of the 290,752 patients that underwent spinal fusion procedures, 374 had the diagnosis of obesity $(\mathrm{OB})$ :

Mean mortality rate of patients was significantly increased (1.9\%, OB vs. $0.6 \%$, no OB $\mathrm{p}=0.01)$

LOS in patients with obesity who underwent spinal fusion was significantly increased (7.02 days, OB vs. 4.02 days, no $\mathrm{OB}, \mathrm{p}=0.0001)$

Total hospital charges were significantly increased $(\$ 142,853.68$, OB vs. $\$ 98,294.43$, no OB, $p=0.0001)$

Age at admission was not significant in patients with obesity (58.43 years, OB vs. 57.69 years, no OB, $\mathrm{p}=.307$ )

Conclusion Patients who are obese and undergo a spinal fusion procedure suffer from increased mortality rate, LOS, and total hospital charges. This study aims to provide physicians with information in the management of patients with obesity that undergoes spinal fusion procedures. Peri-procedural patient optimization could provide a potential avenue to lower LOS, total in-hospital charges, and mortality in patients. Disclosures T. Colburn: None. D. Schirmer: None. Z. RoseReneau: None. B. Wright: None. 